\title{
Anti-inflammatory effects of grape seed extract in hemodialysis patients; a pilot study
}

\author{
Amir Hesam Alirezaei ${ }^{1}$, Alireza Shirzadeh Barough ${ }^{1 *}$, Tabassom Azizi ${ }^{1}$, Siavash Shirzadeh Barough ${ }^{1}$, \\ Amir Ghorbanihaghjo ${ }^{2}$, Nadereh Rashtchizadeh ${ }^{3}$, Hossein Babaie ${ }^{2}$, Abbas Delazar ${ }^{2}$
}

${ }^{1}$ Department of Nephrology, Modarres Hospital, Shahid Beheshti University of Medical Sciences, Tehran, Iran
${ }^{2}$ Biochemistry Lab, Drug Applied Research Center, Tabriz University of Medical Siences, Tabriz, Iran
${ }^{3}$ Biomedical Section, Medical Faculty, Tabriz University of medical Siences, Tabriz, Iran

\section{A R T I C L E I N F O}

Article Type:
Original

\section{Article History:}

Received: 12 October 2016

Accepted: 30 November 2016

Published online: 16 December 2016

\section{Keywords:}

End-stage renal disease

Grape seed extract

Hemodialysis

Inflammation

\begin{abstract}
A B S T R A C T
Introduction: Immune responses and inflammation are major risk factors for mortality in patients with end-stage renal disease (ESRD). Increased oxidative stress is a common feature of ESRD, and it has been speculated that it is interrelated to inflammation. In many studies it has been proved that red grape seed extract (RGSE) provides an antioxidant efficacy.

Objectives: The aim of this study was to investigate the anti-inflammatory effects of RGSE in hemodialysis patients.

Patients and Methods: Thirty hemodialysis individuals were divided into two groups that received either $200 \mathrm{mg} /$ day of the RGSE or placebo for one month. Mean \pm SD of age of patients was $58.5 \pm 10.5$ years. Duration of dialysis was $4 \pm 3.4$ years and 27 patients were male and 3 patients were female. After a 2-week washout period, the groups were crossed over for another one month. Markers of inflammation, lipid profiles and blood factors were measured at baseline after intervention with RGSE or placebo.

Results: Our results showed that RGSE consumption could not make statistically significant changes in markers of inflammation, lipid profiles and blood factors in hemodialysis patients. Conclusion: Although RGSE could not improve markers of inflammation, lipid profiles and blood factors in hemodialysis subjects over a 1 month period. However, this result may be due to short period of treatment or low dose of RGSE. Further studies need to clear these contradictions.
\end{abstract}

\section{Implication for health policy/practice/research/medical education:}

In a study on 30 hemodialysis patients, we found, RGSE could not improve markers of inflammation, lipid profiles and blood factors over one month period. However, this result may be due to short period of treatment or low dose of RGSE. Further studies need to clear these contradictions.

Please cite this paper as: Alirezaei AH, Shirzadeh Barough A, Azizi T, Shirzadeh Barough S, Ghorbanihaghjo A, Rashtchizadeh N, Babaie H, Delazar A. Anti-inflammatory effects of grape seed extract in hemodialysis patients; a pilot study. J Renal Inj Prev. 2017;6(3):184-187. DOI: 10.15171/jrip.2017.35.

\section{Introduction}

End-stage renal disease (ESRD) is going to be one of the most important health problems in the world. More than one million patients in 2004 were on treating with renal replacement therapy (RRT). It was estimated that this population will be more than 2 million in 2010 and the cost of treatment will exceed $\$ 1$ trillion $(1,2)$. ESRD patients suffer from inflammation. This increase in inflammatory factors not only worsens their condition, but also increases cardiovascular events and mortality risks. Recent evidence shows that pro-inflammatory cytokines play an important role in creating malnutrition and cardiovascular disease (CVD) (3). In ESRD patients, $\mathrm{C}$-reactive protein (CRP), interleukin 6 (IL-6), interleukin $\beta$ (IL $\beta)$ and tumor necrosis factor (TNF- $\alpha$ ) are increased. Raised oxidative stress is another characteristic of ESRD and is connected with inflammation (4). The level of CRP and pro-inflammatory cytokines like IL $\beta$, IL8, IL1 and TNF- $\alpha$ are prognostic factors for mortality in ESRD population (5). Despite various treatments to control 
of inflammation in ESRD patients, still there is not a certain solution for it. Numerous studies are conducted to find new types of antioxidants in plants. Various plant extracts have been found with antioxidant ingredient and one of these is grape seed. Grape is one of the most commonly consumed fruits in the world. It has different biological functions, due to its rich polyphenol content, most of which are contained in its seeds (60\%-70\%) and its skin (30\%) (6). These ingredients consist of phenolic acids, monomeric, dimeric, trimeric flavanols and polymeric procyanidins. Grape seed proanthocyanidin is the most effective antioxidant (7). It has been reported that polyphenol in grape seeds have several biological activities, including reduction of free radicals, stress oxidative inhibition, antithrombotic and cardioprotective effects $(6,8)$. Several researches have been done to find out antioxidant effect of red grape seed extract (RGSE) on nonsteroidal anti-inflammatory drugs (NSAIDs) induced gastropathy, aminoglycosides induced nephropathy, brain, liver and gastro intestinal mucus of diabetic animals. However, little is known about the anti-inflammatory and antioxidant power of RGSE in dialysis patients.

\section{Objectives}

In this study, we sought to investigate anti-inflammatory impact of RGSE in patients on dialysis patients.

\section{Patients and Methods}

We conducted a placebo-controlled double blind randomized clinical trial to assess the effect of RGSE on blood factors, lipid profile and inflammation in ESRD patients under hemodialysis. Sample size was calculated after consultation with a statistical specialist: 30 patients were enough as it was a pilot study. Therefore, 30 hemodialysis individuals from hospitals related to Shahid Beheshti University in 2013 were recruited.

Exclusion criteria were; malnutrition or cachexia (body mass index $[\mathrm{BMI}]<18.5 \mathrm{~kg} / \mathrm{m}^{2}$, albumin [Alb] $<3 \mathrm{~g} / \mathrm{dL}$ ), hospitalization in recent 3 months, infection (active or chronic), CHF and cirrhosis.

Patients were forbidden from taking statins and vitamin E before the trial starts. All people were treating with erythropoietin and also should be on efficient dialysis (urea reduction more than 60\% after dialysis). After signing informed consent, patients randomized into two groups of 15 receiving either RGSE $200 \mathrm{mg} / \mathrm{d}$ or placebo for 1 month (both RGSE capsule and placebo were produced by Darou Projeh Jaber Company and placebo capsule was contained starch). After a 2-week washout period, groups were crossed over for another 1 month. Blood samples were collected three times; at baseline, after first month and at the end of study. Blood serum was separated and saved in $-20^{\circ} \mathrm{C}$. At the end of the study, samples were assessed for the levels of lipid profile, high-sensitivity CRP (hsCRP), Alb, IL-6 and TNF-a.

\section{Ethical issues}

1) The research followed the tenets of the Declaration of
Helsinki; 2) Informed consent was obtained, and they were free to leave the study at any time; and 3) the research was approved by the ethical committee of Shahid-Beheshti University of Medical Science (\#SBMU.REC.1392.364).

\section{Statistical analysis}

Data were gathered in a questionnaire and were analyzed by SPSS software (version 18.0, SPSS Inc., Chicago, USA). The results of quantitative variables are presented as mean \pm standard deviation (SD). One-way analysis of variance (ANOVA) was applied and $P$ values less than 0.05 were considered significant.

\section{Results}

A total of 30 hemodialysis patients were recruited and randomly assigned to RGSE and placebo groups. Patients were divided into two groups that received either 200 $\mathrm{mg} / \mathrm{d}$ of the RGSE or placebo for 1 month. Mean \pm SD of age of patients was $58.5 \pm 10.5$ years. Duration of dialysis was $4 \pm 3.4$ years and 27 patients were male and 3 patients were female.

Table 1 shows levels of blood factors, lipid profile and markers of inflammation in our 30 patients. Data analysis demonstrated that RGSE consumption for 1 month could not make statistically significant changes in these markers (Table 1).

\section{Discussion}

ESRD is going to be one of the most important health problems in the world. It is estimated that around 52000 patients will be on RRT by the end of 2015 in Iran. A common problem in ESRD patients is inflammation which is a risk factor for cardiovascular complications and death. Free radicals play an important role in the pathophysiology of different diseases such as cardiovascular, nephropathy and ischemia. Free radicals and oxidative stress are the main mediators of apoptosis by producing lipid peroxidase and lipid hydroperoxidase (9). CRP level, IL $\beta$, IL8, IL1 and TNF- $\alpha$ are prognostic factors for mortality in ESRD population. It has not been found a certain solution to control of inflammation in ESRD and dialysis patients yet. Studies show that many plant extracts have polyphenolic flavonoids with antioxidant activity; among them silymarin, catechin and quercetin have protective effect on experimental diabetes by increasing antioxidant enzymes activities (10). One of these plants with diverse antioxidant action is grape seed. Its ingredients consist of phenolic acids (gallic acid and ellagic acid), monomeric flavanols (catechin and epicatechin), dimeric, trimeric and polymeric procyanidins (11). Proanthocyanidins are a class of polyphenols found in grape seed. Chemically, they are oligomeric flavonoid. Grape seed proanthocyanidin is the most effective antioxidant. It has a wide spectrum biological, pharmacological, therapeutic and chemoprotective effects against free oxygen radicals and oxidative stress $(7,12)$. In a study, in 2009 , anti-inflammatory effect of grape seed extract (GSE) in Alzheimer disease mice were investigated. Polyphenol derived from GSE prevented 
Table 1. Levels of markers of inflammation, blood factors and lipid profile at baseline, after receiving placebo and after treatment with RGSE

\begin{tabular}{|c|c|c|c|c|}
\hline & Baseline & Placebo & Drug & $P$ value \\
\hline hsCRP (mg/L) & $10.07 \pm 9.46$ & $12.19 \pm 11.7$ & $12.95 \pm 10.64$ & 0.15 \\
\hline $\mathrm{ESR}(\mathrm{mm} / \mathrm{h})$ & $27.45 \pm 14.49$ & $28.24 \pm 15.86$ & $29.14 \pm 18.02$ & 0.60 \\
\hline Alb (g/dL) & $4.12 \pm 0.52$ & $4.25 \pm 0.61$ & $4.22 \pm 0.62$ & 0.29 \\
\hline IL-6 (mg/dL) & $33.23 \pm 11.98$ & $36.80 \pm 14.29$ & $32.77 \pm 11.80$ & 0.34 \\
\hline TNF- $\alpha$ (mg/ dL) & $23.70 \pm 9.64$ & $22.90 \pm 9.36$ & $21.72 \pm 5.65$ & 0.55 \\
\hline VLDL (mg/dL) & $29.90 \pm 11.11$ & $28.17 \pm 11.01$ & $29.14 \pm 13.81$ & 0.72 \\
\hline Cholesterol (mg/ dL) & $137 \pm 24.72$ & $144 \pm 27.7$ & $140.2 \pm 30.45$ & 0.22 \\
\hline LDL-C (mg/dL) & $70 \pm 21.77$ & $72.67 \pm 26.82$ & $72.40 \pm 23.7$ & 0.57 \\
\hline $\mathrm{HDL}-\mathrm{C}(\mathrm{mg} / \mathrm{dL})$ & $32.62 \pm 9.24$ & $34.69 \pm 9.53$ & $32.07 \pm 8.88$ & 0.29 \\
\hline $\mathrm{TG}(\mathrm{mg} / \mathrm{dL})$ & $172 \pm 88.46$ & $158 \pm 98.07$ & $168.8 \pm 90.3$ & 0.44 \\
\hline PTH ( $\mathrm{gg} / \mathrm{mL})$ & $383 \pm 375$ & $327 \pm 301$ & $344 \pm 364$ & 0.43 \\
\hline Calcium (mg/dL) & $8.67 \pm 0.47$ & $8.74 \pm 0.70$ & $8.54 \pm 0.62$ & 0.35 \\
\hline Phosphorus (mg/dL) & $5.24 \pm 1.52$ & $5.33 \pm 0.37$ & $5.54 \pm 1.41$ & 0.41 \\
\hline Hemoglobin (g/dL) & $11.17 \pm 1.51$ & $10.85 \pm 1.52$ & $10.98 \pm 1.54$ & 0.26 \\
\hline
\end{tabular}

Abbreviations: hsCRP, high-sensitivity CRP; Alb, albumin; TNF- $\alpha$, tumor necrosis factor; LDL-C,low-density lipoprotein cholesterol;TG, triglyceride; HDL-C, high-density lipoprotein cholesterol; VLDL, very LDL; PTH, parathyroid hormone; IL-6, interleukin 6.

Data are presented as mean \pm SD. Repeated measure ANOVA was used to compare different treatment groups.

amyloid beta aggregation and decreased inflammation in their brain (13). In our study 30 hemodialysis patients who treated by receiving either placebo or GSE. After 1 month, GSE did not make a significant change on markers of inflammation and blood factors. Influence of GSE on hyperlipidemia and oxidized low-density lipoprotein cholesterol (LDL-C) was investigated in a study in 2012. Its results showed a significant decline in total cholesterol, LDL-C and oxidized LDL (OxLDL). Also triglyceride (TG) and very LDL-C (VLDL-C) were reduced while high-density lipoprotein cholesterol (HDL-C) raised but not significant. The differences between these results with our results possibly is due to our shorter treatment duration (14). Another study focused on endothelial dysfunction, oxidative stress, and inflammation and insulin resistance in the type 2 diabetes patients over a 4-week period. At the end of the study fructosamine, whole blood glutathione, total cholesterol and CRP had significant changes. This study was in contrast with our results and may be due to lower administered doses of GSE in our patients (15). Moreover, Ulusoy et al showed that GSE prevents cyclosporine induced nephropathy. This ameliorative impact may be due to anti-oxidant and anti-apoptotic influence of GSE. Additionally, another study showed that, GSE improved kidney function (16). Likewise, Safa et al detected the protective effect of GSE on gentamicin induced nephropathy in rats, which was proved by its beneficial effects on histologic features of injury induced by gentamicin (11).

\section{Conclusion}

Although GSE did not improve markers of inflammation, lipid profiles and blood factors in hemodialysis subjects over 1 month period, various studies have mentioned some beneficial effects. This could be due to short period of treatment or low dose of GSE. Further works need to clear these contradictions.

Limitations of this study

Although the research has reached its aims, there were some unavoidable limitations. Firstly, time limitation and secondly, small proportion of the patients were the main limitations of our study.

\section{Acknowledgments}

This research was supported by Urology \& Nephrology Research Center of Shahid Beheshti University of Medical Sciences, Tehran, Iran. We thank our colleagues from Shahid Beheshti Medical School who provided insight and expertise and greatly assisted the research, although they may not agree with all of the conclusions of this paper.

Authors' contribution

All authors contributed equally to the manuscript.

\section{Conflicts of interest}

The authors declared no competing interests.

\section{Ethical considerations}

Ethical issues (including plagiarism, data fabrication, double publication) have been completely observed by the authors.

\section{Funding/Support}

Urology \& Nephrology Research Center of Shahid Beheshti University of Medical Sciences, Tehran, Iran.

\section{References}

1. Lysaght MJ. Maintenance dialysis population dynamics: current trends and long-term implications. J Am Soc Nephrol. 2002;13 Suppl 1:S37-40.

2. Mahdavi-Mazdeh M, Heidary Rouchi A, Norouzi S, 
Aghighi M, Rajolani H, Ahrabi S. Renal replacement therapy in Iran. Urol J. 2007;4:66-70.

3. Stenvinkel P. Inflammation in end-stage renal failure: could it be treated? Nephrol Dial Transplant. 2002;17 suppl 8:338. doi: 10.1093/ndt/17.suppl_8.33

4. Maruyama Y, Lindholm B, Stenvinkel P. Inflammation and oxidative stress in ESRD--the role of myeloperoxidase. J Nephrol. 2004;17 Suppl 8:S72-6.

5. Stenvinkel P, Lindholm B. C-reactive protein in end-stage renal disease: are there reasons to measure it? Blood Purif. 2005;23:72-8. doi:10.1159/000082014

6. Park SH, Park TS, Cha YS. Grape seed extract (Vitis vinifera) partially reverses high fat diet-induced obesity in C57BL/6J mice. Nutr Res Pract. 2008;2:227-33. doi: 10.4162/nrp.2008.2.4.227.

7. Yilmaz Y, Toledo RT. Health aspects of functional grape seed constituents. Trends Food Sci Technol. 2004;15:42233. doi:10.1016/j.tifs.2004.04.006

8. Sano T, Oda E, Yamashita T, Naemura A, Ijiri Y, Yamakoshi J, et al. Anti-thrombotic effect of proanthocyanidin, a purified ingredient of grape seed. Thromb Res. 2005;115:115-21. doi:10.1016/j.thromres.2004.07.015

9. Bagchi D, Sen CK, Ray SD, Das DK, Bagchi M, Preuss HG, et al. Molecular mechanisms of cardioprotection by a novel grape seed proanthocyanidin extract. Mutat. 2003;523:8797.

10. Sabu MC, Smitha K, Kuttan R. Anti-diabetic activity of green tea polyphenols and their role in reducing oxidative stress in experimental diabetes. J Ethnopharmacol. 2002 30;83:109-16.

11. Safa J, Argani H, Bastani B, Nezami N, Ardebili BR,
Ghorbanihaghjo Aet al. Protective effect of grape seed extract on gentamicin-induced acute kidney injury. Iran J Kidney Dis. 2010;4:285.

12. Bagchi D, Garg A, Krohn RL, Bagchi M, Bagchi DJ, Balmoori J, et al. Protective effects of grape seed proanthocyanidins and selected antioxidants against TPA-induced hepatic and brain lipid peroxidation and DNA fragmentation, and peritoneal macrophage activation in mice. Gen Pharmacol. 1998;30:771-6.

13. Wang YJ, Thomas P, Zhong JH, Bi FF, Kosaraju S, Pollard $A$, et al. Consumption of grape seed extract prevents amyloid- $\beta$ deposition and attenuates inflammation in brain of an Alzheimer's disease mouse. Neurotox Res. 2009;15:314. doi: 10.1007/s12640-009-9000-x.

14. Razavi SM, Gholamin S, Eskandari A, Mohsenian N, Ghorbanihaghjo A, Delazar A, et al. Red grape seed extract improves lipid profiles and decreases oxidized low-density lipoprotein in patients with mild hyperlipidemia. J Med Food. 2013;16:255-8. doi: 10.1089/jmf.2012.2408.

15. Kar P, Laight D, Rooprai HK, Shaw KM, Cummings M. Effects of grape seed extract in type 2 diabetic subjects at high cardiovascular risk: a double blind randomized placebo controlled trial examining metabolic markers, vascular tone, inflammation, oxidative stress and insulin sensitivity. Diabet Med. 2009;26:526-31. doi: 10.1111/j.1464-5491.2009.02727.x.

16. Ulusoy S, Ozkan G, Yucesan FB, ERSÖZ Ş, Orem A, Alkanat $\mathrm{M}$, et al. Anti-apoptotic and anti-oxidant effects of grape seed proanthocyanidin extract in preventing cyclosporine A-induced nephropathy. Nephrology (Carlton). 2012;17:372-9. doi: 10.1111/j.1440-1797.2012.01565.x.

Copyright (C) 2017 The Author(s); Published by Nickan Research Institute. This is an open-access article distributed under the terms of the Creative Commons Attribution License (http://creativecommons.org/licenses/by/4.0), which permits unrestricted use, distribution, and reproduction in any medium, provided the original work is properly cited. 